\title{
Review
}

\section{Advances in Studies on Prevalence and Interaction Mechanism of Acquired Immunodeficiency Syndrome and Tuberculosis Coinfection}

\author{
Chao Meng, Na Li, Zhaoxiao Tong, Huangxin Yan, Shenxiao Min \\ Clinical Laboratory, The Tianjin Second People's Hospital, Tianjin China
}

Keywords

AIDS; TB; Coinfection; Interaction mechanism;

Sum up

\section{Correspondence}

Shenxiao Min,

E-mail: shenxiaomin420@163.com

DOI: 10.1515/ii-2017-0141

\begin{abstract}
Human immunodeficiency virus (HIV) and tuberculosis (TB) coinfection is a serious public health problem. HIV and TB promote each other, accelerating development of HIV to acquired immunodeficiency syndrome (AIDS) and heightening TB mortality. Determining interaction mechanism between HIV and Mycobacterium tuberculosis can lead to development of effective treatments. This study summarizes prevalence status of AIDS and $\mathrm{TB}$ coinfection and research advances concerning their interaction mechanism.
\end{abstract}

Acquired immunodeficiency syndrome (AIDS) is a disease characterized by retrovirus injury of CD4+ cells and causes incompetent cellular immune function. Tuberculosis (TB) is chronic infectious disease caused by Mycobacterium tuberculosis (Mtb) infection. Mtb invades various organs of the body and mainly infects lungs. TB is also called phthisis and white plague, which was an ancient infectious disease. TB is the common and early occurring opportunistic infection (occupies 20\% to 50\%) of AIDS. Morbidity of AIDS-infected patient with TB is 30 to 50 times that of normal individuals. Patient conditions may rapidly deteriorate because of coinfection of AIDS and TB and mutual promotion of these diseases.

\section{Epidemic Status of Coinfection of AIDS and TB}

\section{Epidemic Status of TB}

In 1990, the number of newly infected TB patients reached 6.6 million globally. In 2000, the number increased to 8.3 million rose to 9.27 million in 2007. TB patients mainly came from Asia (55\%) and Africa (31\%), followed by the Middle East (6\%), Europe (5\%), and America (3\%). The top five countries affected by TB include India ( 2 million cases), China (1.3 million cases), Indonesia (530,000 cases),
Nigeria (460,000 cases), and South Africa $\left(460,000\right.$ cases) ${ }^{[1]}$. According to statistics of the World Health Organization (WHO), existing TB patients approximately reach 20 million. Approximately 3.8 million TB patients die, and 8 million individuals are infected per year ${ }^{[2]}$.

China is one of the 22 countries that are severely infected by $\mathrm{TB}$ and features the second highest number of patients in the world after India ${ }^{[2]}$. From 2001 to 2010, notification cases of TB were constantly the highest among infectious diseases in China. In reference to fifth epidemiological investigation in 2010 , morbidity of active TB totals $392 / 100,000$ population, whereas morbidity of infectious TB reaches $100 / 100,000$ population. These findings indicate that an average of 400 active TB patients exist out of 100,000 people in China. A quarter of these patients are infectious. Accordingly, in 2010, the estimated total number of active TB in China totaled 5.23 million, and infectious TB accounted for 1.34 million cases $^{[3]}$.

\section{Epidemic Status of AIDS}

In 1982, the American Centers for Disease Control and Prevention (CDC) officially named the disease as "Acquired Immune Deficiency Syndrome”. In 1983, the first human immunodeficiency virus (HIV) was successfully isolated and identified as pathogen of AIDS. 
The WHO statistics indicated that in 2009, the total number of HIV/AIDS infections in the world reached 39.5 million, almost reaching the growth of 15,000 cases per day ${ }^{[4]}$. Up to $95 \%$ of these cases came from developing countries. SubSaharan Africa, South Asia, and Southeast Asia will become the main endemic areas. Thailand, India, and Myanmar showed the highest prevalence of HIV infection in South Asia, whereas Cambodia and Vietnam featured the fastest growing rate in recent years ${ }^{[5]}$. Some reports showed that HIV spreads at a rapid rate in certain areas; when effective treatment measures are undertaken, accumulative number of cases reach four times that of today ${ }^{[6]}$. Among HIV(+) patients, less than 5\% become AIDS-infected patients within two years. Up to $20 \%$ to $25 \%$ become AIDSinfected patients within six years, and 50\% turn into AIDSinfected patients within 10 years.

\section{Epidemic Status of HIV/Mtb Coinfection}

When $\mathrm{HIV}(+)$ individuals are infected by $\mathrm{Mtb}$, then morbidity of TB is 30 times higher compared with the rate of HIV(-) individuals. This condition mainly affects people aged 25 to 44; this age group is also a high-risk group of HIV infection. In 2007, 9.27 million cases of TB infection were reported, and 1.37 million (15\%) cases were HIV-positive. Up to $79 \%$ of cases were in obtained from Africa, and another $11 \%$ were discovered in Southeast Asia ${ }^{[1]}$.

In developing countries, $25 \%$ to $65 \%$ of HIV/AIDS cases present TB. In China, current situations of AIDS and TB are severe. In reference to document ${ }^{[7]}$, among 482 AIDSinfected patients in six regions of this country, TB accounts for $15.9 \%$, whereas the rate for Shanghai reaches $45.4 \%$, Xinjiang $28.3 \%$, and that of Yunnan is $16.7 \%$. TB patients also feature high HIV infection rate. Statistical results of 15 developing countries displayed $17 \%-49 \%$ and $44 \%-$ $77 \%$ HIV infection rates of patients with TB and those with extra pulmonary $\mathrm{TB}$, respectively ${ }^{[8]}$. HIV/Mtb coinfection promotes both diseases. This condition causes difficulties in controlling TB and AIDS infection. Globally, 9\% of new TBinfected cases are caused by HIV infection ${ }^{[9]}$. In sub-Saharan areas, $31 \%$ of new TB-infected cases are caused by HIV ${ }^{[10]}$.

$\mathrm{HIV} / \mathrm{Mtb}$ coinfection drew extensive attention among various countries in the world. Global HIV/Mtb coinfection cases increased at a rate of $10 \%$ per year since 1990 . Up to $70 \%$ of patients originate from sub-Saharan Africa and Western Pacific areas, whereas the rate of coinfection in Africa areas exceeds $80 \%{ }^{[11][12]}$. Multidrug resistance was also observed with HIV/Mtb coinfection ${ }^{[13]}$.

Prevalence of HIV causes high TB mortality. In accordance to WHO statistics in 2009, the number of AIDSrelated deaths reached 1.8 million, a quarter of which was caused by TB. In 2000, $11 \%$ of AIDS-infected patients died of TB. To date, at least 14 million patients present HIV/Mtb co-infection. As the most common opportunistic infectious disease, TB is the most common cause of death in AIDS patients $^{[14][15]}$.

\section{Interaction of AIDS and TB}

HIV/AIDS and Mtb spread rapidly in the world, especially in developing countries. HIV invades and destroys the human immune system, increasing susceptibility of the body to opportunistic infections. Mtb is the most common opportunistic infectious pathogen. HIV and TB interact with each other. Globally, 30\% of HIV deaths results from Mtb infection ${ }^{[2]}$. Some studies reported that high mortality after HIV and Mtb coinfection possibly results from activation of innate and adaptive immunity of organisms by $\mathrm{Mtb}$, generation of cytokines, and intracellular signal transduction that induces HIV replication. HIV replication leads to increased viral load, large destruction of $C D+T$ lymphocytes, and high mortality rate of coinfection ${ }^{[16]}$.

\section{Effects of HIV to Mtb Infection}

Risk of HIV TB infection increases by 20 times from unapparent infection to active $\mathrm{TB}^{[17]}$. $\mathrm{HIV}(-) \mathrm{TB}$ patients feature $10 \%$ chance of developing active $\mathrm{TB}$ in their lifetime, whereas $\operatorname{HIV}(+)$ patients possess $10 \%$ chance of developing active TB in just one year and $60 \%$ chance in their lifetime. HIV infection is the most important risk factor of Mtb infection; it can lead to TB endogenous recrudescence, exogenous reinfection, and primary infection. HIV is a retrovirus characterized by reverse transcription replication in T4 cells, damaging CD4+ cells. Damage mechanism is as follows: (1) when HIV enters the human body, it selectively infects T4 lymphocytes, ruptures cell, and causes progressive decrease in T4 lymphocytes. Human anti-TB is mainly mediated by $\mathrm{T}$ lymphocytes and macrophages (cytoimmunologic monitoring plays a leading role). Therefore, ability of macrophages to inhibit Mtb and form granuloma decreases. 2) With progression of AIDS, antibody-dependent cytotoxicity, which is mediated by gp120+ antigen complex for HIV outer membrane, can also 
impair CD4+ cells and decrease its functions and number. 3) Studies showed that HIV affects generation of interleukin (IL)-2 and reduces interferon gamma (IFN- $\gamma$ ). Thus, cell resistance to $\mathrm{Mtb}$ decreases, considering the ruptured balance between the body and Mtb. 4) HIV damages bone marrow stem cells, inhibits formation of CD4 cells, and thus weakens the immune system ${ }^{[18]}$; 5) Secretion of Th2 cytokines increases in plasma of HIV-infected patients. Th2 cytokines can inhibit immune function, inducing susceptibility of the body to Mtb. 6) Finally, deletion of Mtbspecific CD4+ cells is a key factor for susceptibility to Mtb after HIV infection ${ }^{[19]}$.

\section{Effect of Mtb to HIV Infection}

In 1996, the WHO CDC in the United States considered TB and extra pulmonary TB as "directional disease" for $\mathrm{HIV}(+)$ to become $\operatorname{AIDS}^{[20]}$. Mtb is first engulfed by macrophages during Mtb invasion in the human body. Macrophages act as antigens that regulate and activate $\mathrm{T}$ cells (mainly including CD4+ T and CD8+ T cells, of which CD4+ T cells are the most important immune cells), secreting a series of cytokines to activate macrophages. Thus, specific immune responses to $\mathrm{Mtb}$ are generated. Any abnormality occurring at any step during this process causes abnormal immune responses.

Host immune response to Mtb can exacerbate HIV replication and infection of the body. Related mechanisms are as follows: (1) Mtb mainly attacks CD4+ T cells, improving expression of chemokine receptor CXCR4 on peripheral blood CD4+ T lymphocytes. At the same time, Mtb infection activates $\mathrm{T}$ lymphocytes in the body. Cytokines released by lymphocytes significantly increase transcriptional quantity of HIV provirus. Mtb infection increase by 2.7 times monocyte chemoattractant protein-1 (MCP-1), which is secreted by monocytes and macrophages. MCP-1 activates HIV transcription, whereas Tat protein, a HIV transcript, enables MCP-1 expression. In this manner, MCP-1 and HIV promote each other, increasing HIV transcription quantity in TB infection areas and deteriorating diseases; (2) IFN- $\gamma$, IL-2, IL-2, and tumor necrosis factor (TNF)- $\alpha$ is released after Mtb infection. Level of TNF- $\alpha$ in blood of HIV/TB patients is 31 times than those of simple HIV infectors ${ }^{[21]}$. These factors can stimulate repeat sequence of 5 ' long terminal of HIV genome to speed up HIV replication $^{[3]}$, accelerate course of AIDS, and reduce survival rate of HIV-infected patients ${ }^{[22]}$; (3) The main component of Mtb cell wall, lipoarabinomannan, induces HIV replication.
Mtb infection promotes HIV penetration and accelerates replication $^{[23]}$; (4) Mtb itself and pure protein derivatives induce HIV expression in monocytes and rapidly increase P24 content in the body ${ }^{[23]}$; (5) Mtb enhances susceptibility of HIV to CD4+ T cells through Toll-like receptor-2mediated pathway ${ }^{[24]}$; (6) Lastly, Mtb infection and bacillus Calmette-Guérin vaccination may also be an important factor during HIV infection ${ }^{[24]}$.

\section{Perspective}

Emergence and prevalence of AIDS enable resurgence of TB. AIDS and TB promote each other, accelerate development of HIV into AIDS, and increase mortality rate of TB. To date, no effective prevention and treatment measures address HIV/Mtb coinfection. The interaction between HIV and MTB should first be clarified to determine effective therapeutic measures. To improve survival rate and quality of life of patients, we should strengthen collaboration, timely identify HIV and Mtb patients using existing technology, and actively prevent and treat these diseases.

\section{Declarations}

\section{Acknowledgements}

No.

\section{Competing interests}

The authors declare that they have no competing interest.

\section{Authors' contributions}

C Meng and N Li made the literature analysis and wrote, discussed and revised the manuscript of this review. ZX Tong, HX Yan and SX Min critically analyzed and corrected the manuscript. All authors read and approved the final manuscript.

\section{References}

1 World Health Organization. Global tuberculosis control: epidemiology, strategy, financing: WHO report. Geneva: World Health Organization, 2009:411.

2 World Health Organization, WHO report 2007global tuberculosis control surveillance, planning. Financing. Geneva: WHO Press, 2007:270.

3 Tang Shenjie, Xiao Heping. "Trend of Tuberculosis and Future Prospect” 
[J]. Chinese Journal of Practical Internal Medicine, 2012, 32(8):565-8.

4 McElrath MJ, Haynes BF. Induction of immunity to human immunodeficiency virus type-1by vaccination. Immunity, 2010, 33(4):542-54.

5 Lawn SD, Harries AD, Anglaret X, et al. Early mortality among adults accessing antiretroviral treatment programmes in sub-Saharan Africa. AIDS, 2008, 22(15):1897-908.

6 Qin H. Research Progress in Etiopathology of Aids Complicated with Tuberculosis. Journal of Guangxi, 2011, 27(3):2.

7 Xu Y, Pang X. Process in the Research of Double Infection with Aids and Tuberculosis. Tianjin Medical Journal, 2007, 35(5):393-5.

8 World Health Organization. Global tuberculosis control: WHO Report 2010[EB/OL]. (2010-07-31)[2011-05-31].

9 Xiang X, Li X, Xu K. Aids Therapeutics. Hefei: Anhui Science and Technology Press, 2007: 372

10 Lawn SD, Harries AD, Meintjes G, et al. Reducing deaths from tuberculosis in antiretroviral treatment programmes in sub-Saharan Africa. AIDS, 2012, 26(17):2121-33.

11 Thayil SM, Ho YC, Bollinger RC, et al. Mycobacterium tuberculosis complex enhances susceptibility of CD4 T cells to HIV through a TLR2mediated pathway. PLoS One, 2012, 7(7):e41093.

12 Powell R, Barengolts D, Mayr L, et al. The evolution of HIV-1Diversity in Rural Cameroon and its implications in vaccine design and trials. Viruses. 2010, 2(2):639-54.

13 Jones KD, Hesketh T, Yudkin J. Extensively drug-resistant tuberculosis in sub-Saharan Africa: an emerging public-health concern. Trans R Soc Trop Med Hyg, 2008, 102(3):219-24.

14 UNAIDS (2007) TB-HIV: Networking for Policy Change. A Participant' s Guide, http://wwwwhoint/tb/hiv/en/.
15 World Health Organization. Global Tuberculosis control: WHO Report 2010(2010-07-31) [2011-05-31].

16 Raghavan S, Alagarasu K, Selvaraj P. Immunogenetics of HIV and HIV associated tuberculosis.Tuberculosis (Edinb), 2012, 92(1):18-30.

17 Geldmacher C, Zumla A, Hoelscher M. Interaction between HIV and Mycobacterium tuberculosis: HIV-1-induced CD4T-cell depletion and the development of active tuberculosis. Curr Opin HIV AIDS, 2012,7(3):268-75.

18 Rappuoli R, Aderem A. A 2020vision for vaccines against HIV, tuberculosis and malaria. Nature, 2011, 473(7348): 463-9.

19 Sharma SK, Mohan A, Kadhiravan T. HIV-TB co-infection: epidemiology, diagnosis \& management. Indian J Med Res, 2005, 121(4):550-67.

20 Claeye F, Jackson T. Project delivery in HIV/AIDS and TB in Southern Africa: the cross-cultural management imperative. J Health Organ Manag, 2011, 25(4):469-86.

21 Onyebujoh PC, Ribeiro I, Whalen CC. Treatment Options for HIVAssociated Tuberculosis. J Infect Dis, 2007, 196(Suppl1):S35-45.

22 Ramana RPV, Rajasekaran S, Raja A. Natural killer cell-mediated cytokine response among HIV-positive south Indians with pulmonary tuberculosis. J Interferon Cytokine Res, 2010, 30(1):33-42.

23 Tang Mianying, Pan Zhaoxia. Clinical Analysis of 50 Cases of HIV / AIDS Complicated with Tuberculosis. The Journal of the Chinese Antituberculosis Association, 2007, 29 (5):452-3.

24 Lee S, An YS, Kim JG, et al. A study on the synthesis of polystyrene-silica nanocomposite particles by soap-free emulsion poly merization using cationic initial or in company with colloidal silica sol solution. Nanosci Nanotechnol, 2009, 9(12):7229-35. 